\title{
Comparison of methods for modelling geomagnetically induced currents
}

\author{
D. H. Boteler ${ }^{1}$ and R. J. Pirjola ${ }^{1,2}$ \\ ${ }^{1}$ Geomagnetic Laboratory, Earth Science Sector, Natural Resources Canada, 2617 Anderson Road, Ottawa, Ontario, \\ K1A 0E7, Canada \\ ${ }^{2}$ Finnish Meteorological Institute, P.O. Box 503, Helsinki, Finland \\ Correspondence to: D. H. Boteler (dboteler@nrcan.gc.ca)
}

Received: 5 June 2014 - Revised: 5 August 2014 - Accepted: 8 August 2014 - Published: 19 September 2014

\begin{abstract}
Assessing the geomagnetic hazard to power systems requires reliable modelling of the geomagnetically induced currents (GIC) produced in the power network. This paper compares the Nodal Admittance Matrix method with the Lehtinen-Pirjola method and shows them to be mathematically equivalent. GIC calculation using the Nodal Admittance Matrix method involves three steps: (1) using the voltage sources in the lines representing the induced geoelectric field to calculate equivalent current sources and summing these to obtain the nodal current sources, (2) performing the inversion of the admittance matrix and multiplying by the nodal current sources to obtain the nodal voltages, (3) using the nodal voltages to determine the currents in the lines and in the ground connections. In the Lehtinen-Pirjola method, steps 2 and 3 of the Nodal Admittance Matrix calculation are combined into one matrix expression. This involves inversion of a more complicated matrix but yields the currents to ground directly from the nodal current sources. To calculate GIC in multiple voltage levels of a power system, it is necessary to model the connections between voltage levels, not just the transmission lines and ground connections considered in traditional GIC modelling. Where GIC flow to ground through both the high-voltage and low-voltage windings of a transformer, they share a common path through the substation grounding resistance. This has been modelled previously by including non-zero, off-diagonal elements in the earthing impedance matrix of the Lehtinen-Pirjola method. However, this situation is more easily handled in both the Nodal Admittance Matrix method and the Lehtinen-Pirjola method by introducing a node at the neutral point.
\end{abstract}

Keywords. Electromagnetics (numerical methods)

\section{Introduction}

Geomagnetic disturbances can have a detrimental influence on the operation of the electric power transmission systems. This was first observed during the Easter storm of 1940, when power systems in the northeast of the US and Canada experienced malfunctions of equipment (Davidson, 1940). Major geomagnetic disturbances in 1958, 1972, 1982, 1989, 2003, and 2006 similarly caused problems for power systems in different parts of the world (Boteler et al., 1998; Bolduc, 2002; Wik et al., 2009). Problems arise because the variations of the magnetic field induce electric currents in the power transmission lines. These geomagnetically induced currents (GIC) flow to ground at substations of the power system, where they cause partial saturation of the power transformers (Molinski, 2002; Kappenman, 2007). This produces increased power consumption and heating of the transformer and distortion of the alternating current (AC) waveform, which leads to a variety of effects on the power system, such as mis-operation of protective relays, voltage stability problems, and, in a worst-case scenario, power blackouts and damage to transformers. In 1859, an extreme magnetic storm created widespread problems for the technology of the time: the telegraph system (Boteler, 2006). There is increased concern that a repeat of such an extreme magnetic storm could cause widespread problems for the supply of electricity. This has prompted renewed efforts to precisely understand the geomagnetic effects on power systems so as to make accurate risk assessments and plans for mitigation.

Assessment of the geomagnetic hazard to power systems requires estimates of the expected size of the geomagnetic field variations and knowledge of the earth's conductivity 
structure in the area of the power system. These are used to calculate the geoelectric fields to which the power system will be exposed. These geoelectric fields are then used as input to a power-system model to calculate the flow of GIC throughout the system and assess the impact on power transformers and the operation of the power system as a whole. Considerable work is being done on the size of the geomagnetic field variations at different latitudes and the earth conductivity structure in different geological regions and their influence on GIC (Zheng et al., 2013). Likewise, work is being done to assess the impact of GIC on power systems (Kappenman, 2010; Jacobson et al., 2014). This paper is concerned with the modelling of GIC.

Two main approaches have been used for modelling GIC. One involves using power-system network calculations, either as a separate programme (Foss and Boteler, 2006; Boteler et al., 2014) or as part of commercial software packages (Overbye et al., 2012). This has been the basis for most of the GIC calculations made by the power industry. The other is the GIC modelling programme, presented by Lehtinen and Pirjola (1985), which has been used extensively in the geophysics community (Thomson et al., 2005; Wik et al., 2008; Caraballo et al., 2013; Demiray et al., 2013; Torta et al., 2012). Most studies so far have concentrated only on modelling GIC in the highest-voltage lines, which, because of their design, have lower resistances and so experience larger GIC. However, to more accurately assess the GIC in a power network, it is necessary to include the GIC flows at lower voltage levels and the flow between multiple voltage levels. Also, for geomagnetic hazard assessments, it is necessary to know that reliable GIC values are obtained regardless of the modelling technique being used.

In this paper, we review the different approaches and examine their use for modelling GIC in multiple voltage levels of a power system. Power-network calculations can be made using the Mesh Impedance Matrix method or the Nodal Admittance Matrix method, with the latter most commonly used because of its greater computational efficiency. Here, we first present the derivation of GIC modelling using the Nodal Admittance Matrix method and the derivation of the Lehtinen-Pirjola method, and show that they are equivalent. We then examine how these methods can be applied to modelling GIC in a network with multiple voltage levels. When modelling GIC in a single high-voltage level, nodes in a network are usually grounded through the transformers at the substations, simplifying the GIC calculations. In contrast, inclusion of multiple voltage levels introduces nodes into the network model that do not have a direct connection to the ground. It is shown how this can be handled in each modelling technique and used to calculate the GIC in different types of transformers.

\section{Circuit representation of a power system}

High-voltage power-transmission networks use three-phase $\mathrm{AC}$ with transformers to convert between voltage levels. The transformer can use either delta-connected windings or Yconnected windings. The delta-connected windings do not have a connection to the ground and do not allow GIC to flow, and so will not be considered further. In Y-connected transformers, the windings for the three phases are connected together at a neutral point, which is connected to ground, as shown in Fig. 1. In normal operation the AC currents in the three phases have the same amplitude but are $120^{\circ}$ out of phase and sum to zero at the neutral point, so there is normally no AC flow from the neutral point to ground. However, during fault conditions, there may be a significant unbalance in $\mathrm{AC}$ or lightning strikes can produce large currents in the lines and transformers, and the neutral-ground connections are there to provide a safe discharge path for these currents. The neutral-ground connections also provide a path for GIC to flow from the transformer windings to ground. A variety of transformer configurations are possible: autotransformers, two-winding Y-connected transformers (designated as Y-Y), or transformers with a $\mathrm{Y}$ connection on the high-voltage side and a delta connection on the low-voltage side (designated as $\mathrm{Y}-\Delta$ ). In some cases (typically lower voltage windings), the neutral point of $Y$ windings may not be connected to the ground, and no GIC will flow in these windings. Similarly, delta windings do not have a connection to the ground and so do not provide a path for GIC to flow. Thus, these circuit considerations are not relevant for GIC studies and we will concentrate on transformer winding configurations that allow the flow of GIC.

Two types of transformer provide a path for GIC and are illustrated in Fig. 1. In two-winding transformers, separate windings are used for the high-voltage and low-voltage sides, while, in an autotransformer, the high-voltage and lowvoltage sides share a winding with the low-voltage connection made part way along the winding. Later in the paper, we consider how to model GIC in both the low-voltage and highvoltage parts of the power system. However, initially, we consider GIC flow only in the high-voltage parts of the system. This makes it easier to understand the basic concepts of GIC modelling and enables easier comparison with the original literature, which dealt with only a single voltage level.

To construct a network model for calculating GIC in a power system, the first step is to note that the impedances of each phase of the power system will be identical and so will experience the same levels of GIC. Thus, we need only make calculations for a single phase. This is often done by combining the parallel paths of the three-phase transmission lines and transformer windings into equivalent combined values by dividing the line resistances and transformer resistances by 3. Currents from all three phases flow through the substation grounding resistance, $R_{\mathrm{G}}$ so this is already the appropriate value to use in the combined circuit. The results of 


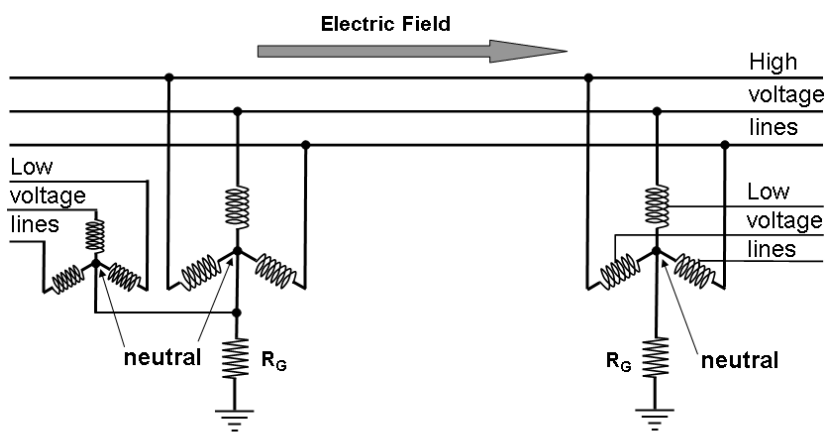

Two winding transformer

Autotransformer

Figure 1. Three-phase transmission lines and substations with a two-winding transformer and an authotransformer.

such modelling will give GIC values that are the sum of the GIC in all three phases, so they have to be divided by 3 to give the GIC per phase. The alternative approach is to make model calculations for a single-phase circuit with resistance values that are all 3 times those in the combined circuit. Thus, the single-phase circuit uses the transmission line resistances and transformer resistances directly and three times the substation grounding resistance. The results in this case give the GIC per phase directly.

Figure 2 shows two single-phase circuits for a power system. The first, shown in Fig. 2a, is an impedance network made up of the resistances of the transmission lines, $r_{\mathrm{L}}$, the resistances of the transformer windings, $r_{\mathrm{T}}$, and connection from the neutral point to the ground, $3 r_{\mathrm{G}}$, where $r_{\mathrm{G}}$ is the actual substation grounding resistance. The driving force for GIC is the electric fields in the power transmission lines (Boteler and Pirjola, 1998), which are represented in the circuit by the voltage source, $e$, equal to the integral of the electric field along the length of the line. An alternative circuit description of the power system is an admittance network, as shown in Fig. 2b. The transformer admittances are given by the inverse of the transformer resistance, $y_{\mathrm{T}}=1 / r_{\mathrm{T}}$, and the admittance of the connection to the ground is $1 / 3 r_{\mathrm{G}}=y_{\mathrm{G}} / 3$. For the electric field in the transmission line, the voltage source, $e$, in series with the transmission line impedance, $r_{\mathrm{L}}$ (Fig. 2a), is converted to the electrically equivalent current source, $j=e / r_{\mathrm{L}}$, in parallel with the transmission line admittance $y_{\mathrm{L}}=1 / r_{\mathrm{L}}$ (Fig. $2 \mathrm{~b}$ ).

GIC calculations can be made using either circuit utilising the Mesh Impedance Matrix method for circuits as in Fig. 2a, and the Nodal Admittance Matrix method and the Lehtinen-Pirjola method for circuits as in Fig. 2b (Boteler, 2014; Lehtinen and Pirjola, 1985). The methods based on the type of circuit shown in Fig. $2 b$ are those most commonly used and will be considered here. Firstly, we present the derivation of the Nodal Admittance Matrix method and the Lehtinen-Pirjola method for calculating GIC and then show that they are mathematically equivalent. Then, we show

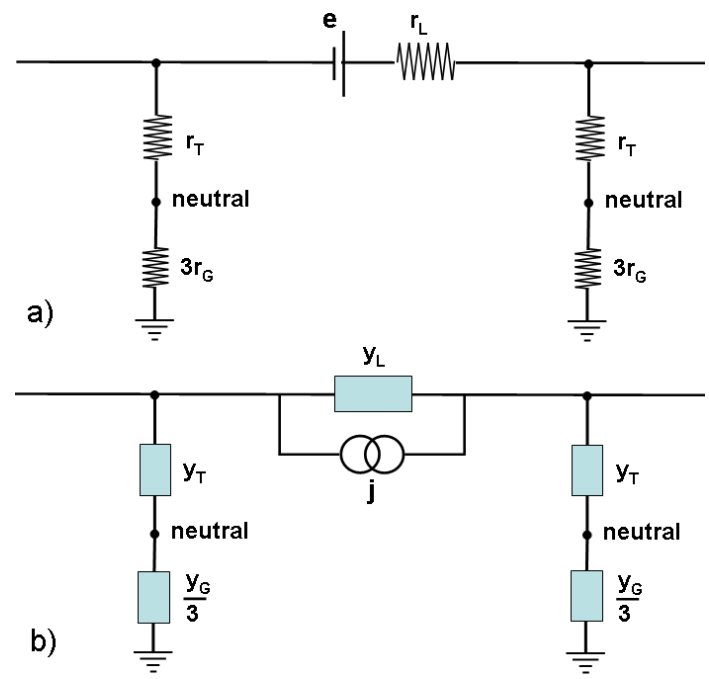

Figure 2. Representation of geomagnetic induction in a power system using: (a) impedance network and voltage sources, and (b) admittance network and current sources.

how these methods can be applied to model GIC in circuits for multiple voltage levels of a power system.

\section{Nodal Admittance Matrix method}

The Nodal Admittance Matrix method uses a circuit model comprised of admittances and current sources, as shown in Fig. 3. Here, the admittances of the transformers and neutralground connection shown in Fig. $2 b$ have been combined to give a single admittance from the high-voltage busbar (node) to the ground at each substation. The admittances between nodes represent the admittance of the transmission lines.

To derive the matrix equations for the network, we start by applying Kirchhoff's current law which states that the algebraic sum of the currents entering any node is zero, i.e. the sum of currents entering on transmission lines equals current flowing to ground. Thus, we can write an equation for any node $i$ of the form

$\sum_{n=1}^{N} i_{n k}=i_{k} \quad n \neq k$,

where $N$ is the number of nodes, $i_{n k}$ is the current from node $n$ to $k$, and $i_{k}$ is the current to the ground from node $k$. This equation also applies if the node is ungrounded, but, in this case, $i_{k}=0$. The current in a line is determined by the current source $j_{n k}$, the voltage difference, $v_{n}-v_{k}$, between nodes at the ends of the line, and the admittance $y_{n k}$ of the line

$i_{n k}=j_{n k}+\left(v_{n}-v_{k}\right) y_{n k}$.

Also summing the current sources directed into each node,

$j_{k}=\sum_{n=1}^{N} j_{n k}$ 


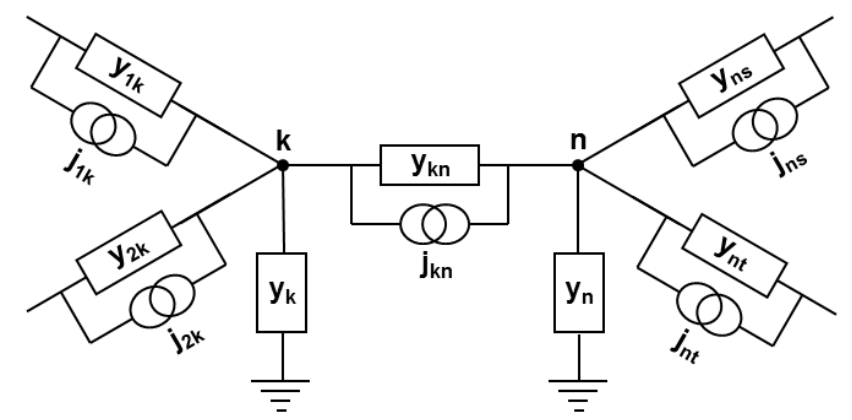

Figure 3. Schematic of admittance network for modelling GIC.

Eq. (1) gives

$j_{k}+\sum_{n=1}^{N}\left(v_{n}-v_{k}\right) y_{n k}=i_{k}$

At the nodes, the nodal voltage $v_{k}$ is related to the current to the ground $i_{k}$ by Ohm's law

$i_{k}=v_{k} y_{k}$

At ungrounded nodes, $y_{k}=0$ so $i_{k}=0$, regardless of the value of $v_{k}$, as mentioned above.

Equations (4) and (5) involve both the nodal voltages, $v_{k}$, and the current to the ground from each node, $i_{k}$, as unknowns. In the Nodal Admittance Matrix method the expression for $i_{k}$, from Eq. (5) is substituted into (4) to give an equation only involving the node voltages $v_{k}$ as the unknowns:

$j_{k}+\sum_{n=1}^{N}\left(v_{n}-v_{k}\right) y_{n k}=v_{k} y_{k}$.

Regrouping terms gives

$j_{k}=v_{k} y_{k}+v_{k} \sum_{n=1}^{N} y_{n k}-\sum_{n=1}^{N} v_{n} y_{n k}$

This can be written in matrix form

$[\mathbf{J}]=[\mathbf{Y}][\mathbf{V}]$,

where $[\mathbf{J}]$ is the current source column matrix with elements

$J_{k}=j_{k}$

and $[\mathbf{Y}]$ is the admittance matrix in which the diagonal elements are the sums of the admittances of all paths connected to node $k$, and the off-diagonal elements are the negative admittances of the connections between nodes $k$ and $n$, i.e.

$Y_{k k}=y_{k}+\sum_{n=1}^{N} y_{n k} \quad n \neq k$,

where we show matrices and matrix elements as upper case to distinguish them from the circuit components in lower case. Remembering that $y_{k}=0$ at ungrounded nodes and noting that the expression for $Y_{k k}$ involves the admittances of branches running from that node, the diagonal elements $Y_{k k}$ in the matrix are non-zero for all nodes, grounded or ungrounded.

The voltages of the nodes are then found by taking the inverse of the admittance matrix and multiplying by the nodal current sources

$[\mathbf{V}]=[\mathbf{Y}]^{-1}[\mathbf{J}]$

These node voltages can then be substituted into Eqs. (2) and (5) to give the currents in the branches and the currents to the ground from each node.

\section{Lehtinen-Pirjola technique}

Matrix equations suitable for determining GIC in a discretely-earthed system were independently derived by Lehtinen and Pirjola (1985), hereafter referred to as LP. As in the Nodal Admittance Matrix method, LP start with Kirchhoff's law for the currents at a nodal point (LP Eq. 8):

$i_{k}=\sum_{n=1}^{N} i_{n k}=-\sum_{n=1}^{N} i_{k n}$,

and relate the current in a line to the driving emf, the voltage difference between the nodes at the ends of the line, and the admittance of the line (LP Eq. 7):

$i_{k n}=y_{k n}\left[e_{k n}+\left(v_{k}-v_{n}\right)\right]$.

Substituting Eq. (13) into (12) gives (LP Eq. 9):

$i_{k}=-\sum_{n=1}^{N} y_{k n}\left[e_{k n}+\left(v_{k}-v_{n}\right)\right]$.

LP also sum the current sources directed into each node (LP Eq. 13). When the path to the ground from each node has zero resistance, the node voltages will be zero and the current in the branches will be exactly equal to current sources. Thus, the sum of the current sources is equal to the current that flows to the ground in this case, and is referred to by LP as "perfect earthing" current:

$J_{k}^{e}=-\sum_{n=1}^{N} e_{k n} y_{k n} \quad n \neq k$

Making this substitution in Eq. (14) gives

$i_{k}=J_{k}^{e}-\sum_{n=1}^{N}\left(v_{k}-v_{n}\right) y_{k n} \quad n \neq k$,

which is the same as that which we obtained earlier in Eq. (4). 
Thus,

$i_{k}=J_{k}^{e}-v_{k} \sum_{n=1}^{N} y_{k n}+\sum_{n=1}^{N} v_{n} y_{k n} \quad n \neq k$.

The first summation represents the dependence of current $i_{k}$ on voltage $v_{k}$, and so gives diagonal elements of a network admittance matrix

$Y_{k k}^{n}=\sum_{n=1}^{N} y_{n k} \quad n \neq k$.

The second summation represents the dependence of current $i_{k}$ on all the other nodal voltages $v_{k}$, and so gives the offdiagonal elements of the network admittance matrix

$Y_{k n}^{n}=-y_{k n} \quad n \neq k$.

Introducing these elements then allows Eq. (17) to be written

$i_{k}=J_{k}^{\mathrm{e}}-\sum_{n=1}^{N} v_{n} Y_{k n}^{n}$

where the summation is now made over all $n$ from 1 to $N$. This is the same as LP Eq. (11). This can be written in matrix form

$\left[\mathbf{I}^{\mathrm{e}}\right]=\left[\mathbf{J}^{\mathrm{e}}\right]-\left[\mathbf{Y}^{n}\right]\left[\mathbf{V}^{n}\right]$

where the elements of column matrix $\left[\mathbf{I}^{\mathrm{e}}\right]$ are the currents $i_{n}$, and the elements of column matrix $\left[\mathbf{V}^{n}\right]$ are the voltages $v_{n}$. It is at this point where the Nodal Admittance Matrix method and the Lehtinen-Pirjola method take different paths. LP make the substitution

$\left[\mathbf{V}^{n}\right]=\left[\mathbf{Z}^{\mathrm{e}}\right]\left[\mathbf{I}^{\mathrm{e}}\right]$

where $\left[\mathbf{Z}^{\mathrm{e}}\right]$ is the earthing impedance matrix. Thus,

$v_{k}=\sum_{n=1}^{N} Z_{k n}^{e} i_{n}$

Substituting Eq. (22) into Eq. (21) gives a matrix equation involving only the node to ground currents $\left[\mathbf{I}^{\mathrm{e}}\right]$ as the unknowns

$\left[\mathbf{I}^{\mathrm{e}}\right]=\left[\mathbf{J}^{\mathrm{e}}\right]-\left[\mathbf{Y}^{n}\right]\left[\mathbf{Z}^{\mathrm{e}}\right]\left[\mathbf{I}^{\mathrm{e}}\right]$.

Gathering terms in $\left[\mathbf{I}^{\mathrm{e}}\right]$ gives

$\left([\mathbf{1}]+\left[\mathbf{Y}^{n}\right]\left[\mathbf{Z}^{\mathrm{e}}\right]\right)\left[\mathbf{I}^{\mathrm{e}}\right]=\left[\mathbf{J}^{\mathrm{e}}\right]$,

where [1] is the unit matrix. Equation (25) can be solved by matrix inversion to give the currents flowing to ground (LP Eq. 12):

$\left[\mathbf{I}^{\mathrm{e}}\right]=\left([\mathbf{1}]+\left[\mathbf{Y}^{n}\right]\left[\mathbf{Z}^{\mathrm{e}}\right]\right)^{-1}\left[\mathbf{J}^{\mathrm{e}}\right]$.

\section{Equivalence of the Lehtinen-Pirjola and Nodal Admittance Matrix methods}

The Lehtinen-Pirjola method and the Nodal Admittance Matrix method are both derived from the same initial equations so it would be expected that they are equivalent. However, with the definitions of the grounding terms that are traditionally used in the two methods, this equivalence only holds under a particular condition, although the definitions can be modified to give a general equivalence. It should also be noted that the network admittance matrix $\left[\mathbf{Y}^{n}\right]$ used by LP is not the same as the admittance matrix [Y] used in the Nodal Admittance Matrix method.

To show the equivalence of the two methods, start with the LP equation:

$\left[\mathbf{J}^{\mathrm{e}}\right]=\left([\mathbf{1}]+\left[\mathbf{Y}^{n}\right]\left[\mathbf{Z}^{\mathrm{e}}\right]\right)\left[\mathbf{I}^{\mathrm{e}}\right]$.

Making the substitution from Eq. (22)

$\left[\mathbf{I}^{\mathrm{e}}\right]=\left[\mathbf{Z}^{\mathrm{e}}\right]^{-1}\left[\mathbf{V}^{n}\right]$

and gives

$\left[\mathbf{J}^{\mathrm{e}}\right]=\left(\left[\mathbf{Z}^{\mathrm{e}}\right]^{-1}+\left[\mathbf{Y}^{n}\right]\right)\left[\mathbf{V}^{n}\right]$.

The earthing impedance matrix defines the voltage between the nodes and a remote earth produced by currents flowing to ground. In the general case, this includes diagonal elements representing the voltage at node $i$, associated with the current flowing to ground from node $i$, and off-diagonal elements representing the voltage produced at node $i$ by currents flowing to ground from other nodes. Such a situation can occur in which current from one node produces a voltage drop in the grounding resistance that affects the voltage at other nodes. This requires the nodes to be very near each other, which means, in practice, that they share the grounding connection at a substation (Pirjola, 2008).

If the earthing current at any node does not affect the voltages at the other nodes, $\left[\mathbf{Z}^{\mathrm{e}}\right]$ becomes diagonal with elements equal to the earthing resistances $r_{i}$ of the nodes. In this case, the inverse of $\left[\mathbf{Z}^{\mathrm{e}}\right]$ is simply the ground admittance matrix $\left[\mathbf{Y}^{\mathrm{e}}\right]$ given by

$Y_{i i}^{e}=y_{i}=1 / r_{i}$

$Y_{i j}^{e}=0 \quad j \neq i$,

and the LP Eq. (29) can be rewritten as an expression for nodal voltages $\left[\mathbf{V}^{n}\right]$.

$\left[\mathbf{J}^{\mathrm{e}}\right]=\left(\left[\mathbf{Y}^{\mathrm{e}}\right]+\left[\mathbf{Y}^{n}\right]\right)\left[\mathbf{V}^{n}\right]$

The $\mathbf{Y}$ terms in brackets are simply separate matrices for the admittance to the ground $\left[\mathbf{Y}^{\mathrm{e}}\right]$ (Eq. 30) and the admittance between nodes [ $\left.\mathbf{Y}^{n}\right]$ (Eqs. 18 and 19). The sum of these two matrices is identical to the Nodal Admittance Matrix [Y] 
which combines both sets of admittances in the one matrix, see Eq. (10). Thus,

$[\mathbf{Y}]=\left[\mathbf{Y}^{\mathrm{e}}\right]+\left[\mathbf{Y}^{n}\right]$.

Combining Eqs. (31) and (32) gives

$\left[\mathbf{J}^{\mathrm{e}}\right]=[\mathbf{Y}]\left[\mathbf{V}^{n}\right]$,

which is the Eq. (8) obtained by the Nodal Admittance Matrix method.

Thus, the LP and Nodal Admittance Matrix methods are shown to be equivalent when the LP earthing impedance matrix has off-diagonal elements equal to zero. When the LP earthing impedance matrix has non-zero, off-diagonal elements an equivalent nodal admittance formulation can be obtained by adopting a definition for the admittance matrix involving $\left[\mathbf{Z}^{\mathrm{e}}\right]^{-1}$ instead of $\left[\mathbf{Y}^{\mathrm{e}}\right]$, which then gives Eq. (29) to be written as Eq. (33).

There are three steps involved in calculating GIC using the Nodal Admittance Matrix method. The first step is to use the voltage sources in the lines representing the induced geoelectric field to calculate equivalent current sources and sum these to obtain the nodal current sources $\left[\mathbf{J}^{\mathrm{e}}\right]$. The second step is to perform the matrix inversion $[\mathbf{Y}]^{-1}$ and multiply by $\left[\mathbf{J}^{\mathrm{e}}\right]$ to obtain the nodal voltages $\left[\mathbf{V}^{n}\right]$. The third step is to use the nodal voltages to determine the currents in the lines and in the ground connections.

In the LP method, the second and third step of the Nodal Admittance Matrix calculation are combined into one matrix expression. This involves inversion of the more complicated matrix in Eq. (25) but yields the currents to the ground $\left[\mathbf{I}^{\mathrm{e}}\right]$ directly from the nodal current sources $\left[\mathbf{J}^{\mathrm{e}}\right]$.

It is also possible to combine all three steps into one matrix expression (Pirjola, 2007). Starting with Eq. (25) and substituting for $\left[\mathbf{I}^{\mathrm{e}}\right]$ from Eq. (28) we obtain

$$
\left[\mathbf{V}^{n}\right]=\left(\left[\mathbf{Z}^{\mathrm{e}}\right]^{-1}+\left[\mathbf{Y}^{n}\right]\right)^{-1}\left[\mathbf{J}^{\mathrm{e}}\right] .
$$

In matrix form, the elements of $\left[\mathbf{J}^{\mathrm{e}}\right]$ (Eq. 15) equal the diagonal elements of the product matrix $[\mathbf{E}]\left[\mathbf{Y}^{n}\right]$, denoted by the $N \times 1$ column matrix $\operatorname{diag}\left(\mathbf{E} \mathbf{Y}^{n}\right)$. Thus, we can write

$$
\left[\mathbf{V}^{n}\right]=\left(\left[\mathbf{Z}^{\mathrm{e}}\right]^{-1}+\left[\mathbf{Y}^{n}\right]\right)^{-1} \operatorname{diag}\left[\mathbf{E} \mathbf{Y}^{n}\right]
$$

Combining this with the relation between the nodal voltages and currents, Eq. (28) gives

$$
\left[\mathbf{I}^{\mathrm{e}}\right]=\left[\mathbf{Z}^{\mathrm{e}}\right]^{-1}\left(\left[\mathbf{Z}^{\mathrm{e}}\right]^{-1}+\left[\mathbf{Y}^{n}\right]\right)^{-1} \operatorname{diag}\left[\mathbf{E} \mathbf{Y}^{n}\right]
$$

The successive steps of calculating these matrix expressions, i.e. calculating $\operatorname{diag}\left(\mathbf{E} \mathbf{Y}^{n}\right)$, performing the matrix inversion, and multiplying by the appropriate admittance matrix, correspond to the three steps described in using the Nodal Admittance Matrix method.

\section{Modelling GIC in multiple voltage levels}

When modelling the GIC flow in multiple voltage levels of a power system, it is also necessary to consider the flow of GIC between voltage levels. The path for GIC flow between voltage levels is through the windings of the transformers at each substation. The type of transformer used determines the nature of the path for flow of GIC. Here, we consider three types of substation: one with only ordinary twowinding transformers, one with only autotransformers, and one with a combination of the two. The details of these three scenarios are presented below, and the next section shows how to model the different scenarios.

\subsection{Two-winding transformers}

In the case of ordinary two-winding transformers, there are separate windings from each voltage busbar (commonly abbreviated as "bus") to the neutral point, as shown in Fig. 4. The neutral point in this case is the junction between the two windings and the path to ground through the grounding resistance of the substation and so has to be included as a node in the network. Thus, the circuit configuration for inclusion in a network model would be as it is shown on the right-hand side of Fig. 4, where windings in parallel have been combined into a single component. The high-voltage (HV) bus and low-voltage (LV) bus are each ungrounded nodes in this network.

\subsection{Autotransformers}

With autotransformers, there is no direct connection between the high-voltage bus and the neutral point. The series winding provides a connection between the high-voltage and lowvoltage buses, and the common (shunt) winding connects the low-voltage bus to the neutral point (Fig. 5). As before, windings in parallel are combined to give the circuit configuration on the right-hand side of Fig. 5. The neutral point is connected to earth through the substation grounding resistance. However, in this case, there is no branching at the neutral point, i.e. any current flowing in the common winding also flows through the grounding resistance, so the grounding resistance and resistance of the common winding can be combined, eliminating the need for a node at the neutral point. Then, the LV bus has a direct connection to the ground through this combined resistance and only the HV bus is represented by an ungrounded node in a network model.

\subsection{Both two-winding transformers and autotransformers}

When two-winding transformers and autotransformers are in use for the same voltage levels at a substation, there are connections between all voltage levels and between those voltage levels and the neutral point, as shown in Fig. 6. The series winding of the autotransformers connect the $\mathrm{HV}$ and 


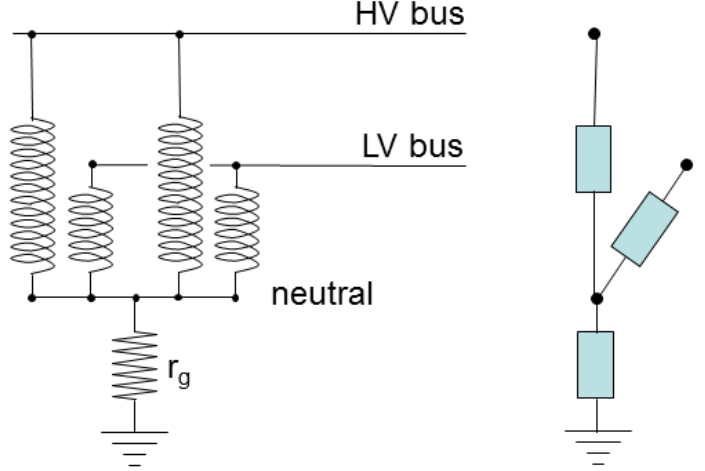

Figure 4. Scenario 1. Substation with two-winding transformers and equivalent admittances for inclusion in the network model.
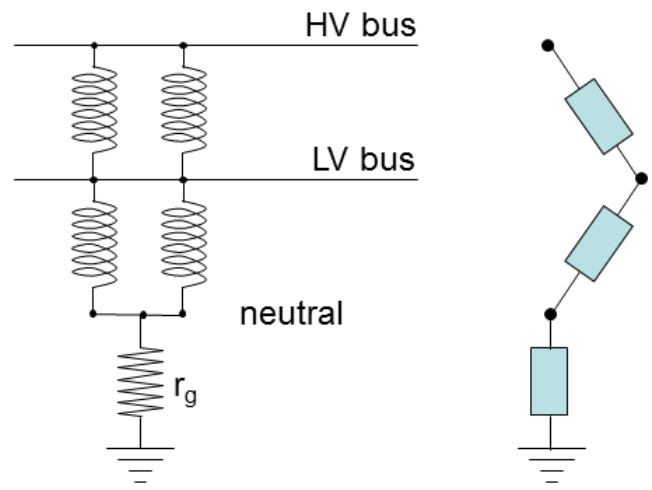

Figure 5. Scenario 2. Substation with autotransformers and equivalent admittances for inclusion in the network model.

LV buses, the HV windings of the two-winding transformers connect the HV bus to the neutral point, and the LV windings of the transformers and common windings of the autotransformers connect the LV bus to the neutral point. As before, all windings in parallel are combined into a single resistance value to give the circuit configuration on the right-hand side of Fig. 6. The neutral point is a branch point between the connections from the HV and LV buses and the connection to the ground and so has to be kept as a node in the network model. Thus, both the HV bus and LV bus are ungrounded nodes in the network model.

\subsection{Modelling the different scenarios}

Scenario 3, with both two-winding transformers and autotransformers, contains all the connections that can occur, so we will develop the equations for this case. The two-winding transformer scenario and the autotransformer scenario can then be derived by deleting the appropriate connection in each case. Consider the simple network shown in Fig. 7. Here, the substation of Scenario 3 is connected to transmission lines that connect to two other substations with only a single path to the ground.
Both Transformers and Autotransformers
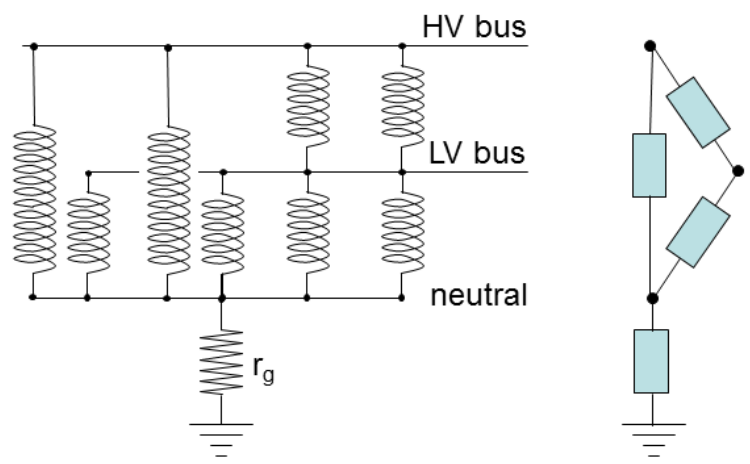

Figure 6. Scenario 3. Substation with both transformers and autotransformers and equivalent admittances for the network model.

Applying Kirchhoff's current law at each node, we equate the sum of currents entering on transmission lines to the current flowing to the ground

$$
\begin{aligned}
-i_{12} & =i_{1}, \\
i_{12}-i_{23}-i_{24} & =0, \\
i_{23}-i_{34}-i_{35} & =0, \\
i_{24}+i_{34} & =i_{4}, \\
i_{35} & =i_{5} .
\end{aligned}
$$

Substituting for the currents in terms of the current sources, nodal voltages, and admittances, and summing the current sources into each node, as in Eq. (7), the equations for each node become

$J_{1}=\left(y_{1}+y_{12}\right) v_{1}-y_{12} v_{2}$,

$J_{2}=-y_{12} v_{1}+\left(y_{12}+y_{23}+y_{24}\right) v_{2}-y_{23} v_{3}-y_{24} v_{4}$,

$J_{3}=-y_{23} v_{2}+\left(y_{23}+y_{34}+y_{35}\right) v_{3}-y_{34} v_{4}-y_{35} v_{5}$,

$J_{4}=-y_{24} v_{2}-y_{34} v_{3}+\left(y_{24}+y_{34}+y_{4}\right) v_{4}$,

$J_{5}=-y_{35} v_{3}+\left(y_{35}+y_{5}\right) v_{5}$,

where, in this example, because no current sources are connected to node $4, J_{4}=0$.

This is an example of the matrix Eq. (8), shown earlier with $[\mathbf{J}]=5 \times 1$ matrix, $[\mathbf{Y}]=5 \times 5$ matrix, $[\mathbf{V}]=5 \times 1$ matrix. The voltages of the nodes are then found by taking the inverse of the admittance matrix and multiplying by the nodal current sources (Eq. 11). These node voltages can then be substituted into Eqs. (2) and (5) to give the network currents. This provides the solution for Scenario 3 with both transformers and autotransformers shown in Fig. 6.

To obtain the solution for Scenario 1 with only a transformer we just need to set $y_{23}=0$ in Eq. (38) and compute a new matrix inversion. Similarly the solution for Scenario 2 with only autotransformers can also be obtained by modifying Eq. (38), in this case setting $y_{24}=0$. In Scenarios 1, 2, 


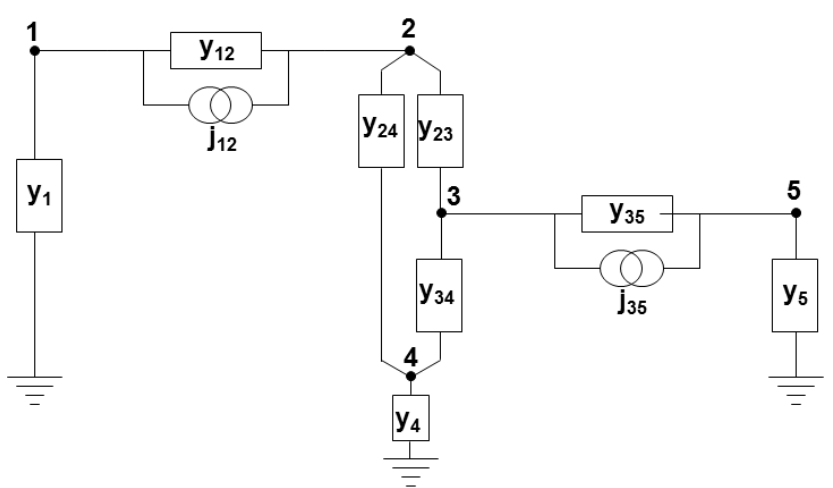

Figure 7. Network model for a substation, as in Scenario 3, connected to transmission lines to substations with a single path to the ground.

and 3 , the connections between nodes 2,3 , and 4 represent the transformer winding resistances at the same substation, so there are no current sources in these branches.

\section{Earthing impedance for a transformer}

In the approach used by Lehtinen and Pirjola (1985), they define an earthing impedance matrix $[\mathbf{Z}]$ that relates the voltages between the nodes and a remote earth to the currents flowing to or from ground at those nodes.

$v_{k}=\sum_{n=1}^{N} z_{k n} i_{n}$

As explained in Sect. 5, if the currents to ground from nodes do not influence the voltages at other nodes, then the earthing impedance matrix is simply a diagonal matrix with diagonal elements equal to the earthing resistances from each node to remote earth. However, if currents from other nodes do affect the voltages, then non-zero, off-diagonal elements occur in the earthing impedance matrix (Lehtinen and Pirjola, 1985).

The earthing impedance matrix provides the link between the nodal voltage and the current flowing to ground from that node. Consider the transformer shown in Fig. 8a. The highvoltage bus is node 1 and the low-voltage bus is node 2 . The high- and low-voltage windings of the transformer have resistances $r_{\mathrm{A}}$ and $r_{\mathrm{B}}$ respectively and both connect to the substation grounding resistance $r_{\mathrm{g}}$.

The relation between current and voltage for each path is given by

$$
\begin{aligned}
& v_{1}=i_{1}\left(r_{\mathrm{A}}+r_{\mathrm{g}}\right)+i_{2} r_{\mathrm{g}}, \\
& v_{2}=i_{2}\left(r_{\mathrm{B}}+r_{\mathrm{g}}\right)+i_{1} r_{\mathrm{g}} .
\end{aligned}
$$
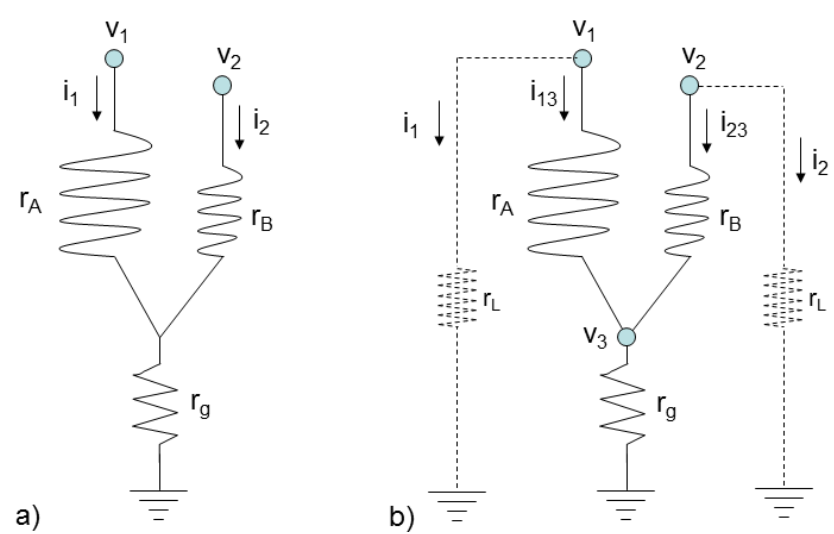

Figure 8. Grounding connection for a transformer. (a) Sharing a path through the grounding resistance and (b) with a node at the neutral point.

This can be written in matrix form:

$\left[\begin{array}{l}v_{1} \\ v_{2}\end{array}\right]=\left[\begin{array}{cc}r_{\mathrm{A}}+r_{\mathrm{g}} & r_{\mathrm{g}} \\ r_{\mathrm{g}} & r_{\mathrm{B}}+r_{\mathrm{g}}\end{array}\right]\left[\begin{array}{l}i_{1} \\ i_{2}\end{array}\right]$.

If $r_{\mathrm{g}}=0$, then the earthing impedance matrix reduces to a diagonal matrix. The inverse of a diagonal matrix is obtained by replacing each element in the diagonal with its reciprocal. If $r_{\mathrm{g}} \neq 0$, then we must use the full formula to find the inverse. The inverse of $[\mathbf{Z}]$ is given by

$[\mathbf{Z}]^{-1}=\left[\begin{array}{cc}\frac{Z_{22}}{|Z|} & \frac{-Z_{12}}{|Z|} \\ \frac{-Z_{21}}{|Z|} & \frac{Z_{11}}{|Z|}\end{array}\right]$,

where the determinant of $\mathbf{Z}$ is $|\mathbf{Z}|=Z_{11} Z_{22}-Z_{12} Z_{21}$.

For the example above, the inverse of $[\mathbf{Z}]$ becomes

$[\mathbf{Z}]^{-1}=\left[\begin{array}{cc}\frac{r_{\mathrm{B}}+r_{\mathrm{g}}}{|Z|} & \frac{-r_{\mathrm{g}}}{|Z|} \\ \frac{-r_{\mathrm{g}}}{|Z|} & \frac{r_{\mathrm{A}}+r_{\mathrm{g}}}{|Z|}\end{array}\right]$,

where $|\mathbf{Z}|=r_{\mathrm{A}} r_{\mathrm{B}}+\left(r_{\mathrm{A}}+r_{\mathrm{B}}\right) r_{\mathrm{g}}$.

This is not a practical formulation to use in the Nodal Admittance Matrix method. In this method, it is better to introduce an extra node at the junction of $r_{\mathrm{A}}, r_{\mathrm{B}}$, and $r_{\mathrm{g}}$, as shown in Fig. 8b. Nodes 1 and 2 are now ungrounded. In the Lehtinen-Pirjola method, this is dealt with by considering a path to the ground for each node with a very high resistance value, $r_{\mathrm{L}}$ (shown as dashed lines in Fig. 8b). The relations between voltages and currents to the ground from the three nodes are now

$v_{1}=i_{1} r_{\mathrm{L}}$,

$v_{2}=i_{2} r_{\mathrm{L}}$,

$v_{3}=i_{3} r_{\mathrm{g}}$.

This can be written in matrix form:

$\left[\begin{array}{l}v_{1} \\ v_{2} \\ v_{3}\end{array}\right]=\left[\begin{array}{ccc}r_{\mathrm{L}} & 0 & 0 \\ 0 & r_{\mathrm{L}} & 0 \\ 0 & 0 & r_{\mathrm{g}}\end{array}\right]\left[\begin{array}{l}i_{1} \\ i_{2} \\ i_{3}\end{array}\right]$. 
This is the earthing impedance matrix and now contains no non-zero, off-diagonal elements. The transformer resistances $r_{\mathrm{A}}$ and $r_{\mathrm{B}}$ are now not part of the earthing impedance matrix but will appear as branches in the network admittance matrix. Because of this, the currents through these resistances are designated as $i_{1}$ and $i_{2}$ in Eqs. (40), (41) and (42) and Fig. 8a, and as $i_{13}$ and $i_{23}$ in Fig. 8b. $i_{1}$ and $i_{2}$ in Eq. (45) and (46) and Fig. 8b are then just the currents through the added connections to the ground and have values near zero because of the high value of $r_{\mathrm{L}}$. This is as it should be, because the ground connections from nodes 1 and 2 in Fig. $8 \mathrm{~b}$ are only added because of the need to have a value in the earthing impedance matrix used in the LP method. The ground connections from nodes 1 and 2 are not needed in the Nodal Admittance Matrix method.

\section{Discussion}

The equations derived for all of the scenarios considered are consistent with the general matrix equations defined in Eqs. (8)-(11). Thus, the general scheme will work for any situation, provided that the right nodes and connections are chosen that accurately represent the circuit scenario being modelled. In Scenarios 1, 2, and 3, we include a node at the neutral points of the transformers. This is needed if there are two-winding transformers at the substation being modelled. In Scenario 2, where there are only autotransformers, the grounding resistance can be combined with the common winding of the autotransformers and the node removed. It is kept in this example for consistency with the other scenarios considered. For simplicity nodes 1 and 5 in Fig. 7, representing other substations are shown with only a single path to the ground. In practice, each of these substations could also be represented by nodes for different voltage levels and the neutral point. Including a node at the neutral point of a substation provides flexibility in the modelling, in that a general scheme can be used for any type of transformer. It has the added advantage that the grounding resistance can be treated separately in the modelling.

The mathematical methodology used here is no different than that described previously. The difference is in how we think about the network. The model is comprised of nodes with branches between nodes and a connection to the ground from the nodes. In traditional modelling, the branches are the transmission lines and the connection to the ground is the path through the transformers and grounding resistance at each substation. Every transmission line is exposed to the geomagnetic induction, so this is represented by a current source in parallel with the admittance of each branch. Now, with the introduction of nodes at the neutral points, the branches include the transmission lines, as before, but also the transformer windings at the substations. There are no current sources associated with the latter branches. The nodes at which the transformer windings connect to the transmission lines have no direct ground connection. The nodes at the neutral points have a ground connection that is simply the grounding resistance of the substation.

Mäkinen (1993) is probably the first publication about a GIC study that precisely considers two voltage levels. Mäkinen uses the Lehtinen-Pirjola technique for the Finnish 400 and $220 \mathrm{kV}$ grids. Mäkinen's approach is also briefly summarised by Pirjola (2005). Because there are nodes that are very close to each other, Mäkinen assigns non-zero values to some off-diagonal elements of the earthing impedance matrix. Recent numerical and theoretical studies, however, show that, if a node is ungrounded, the off-diagonal elements of the earthing impedance matrix associated with this node do not play any role for GIC in the network. Thus, they can be set, for example, equal to zero, as shown in Eqs. (45) and (46). This is actually an observation that also indicates the full equivalence between the Nodal Admittance Matrix method and the Lehtinen-Pirjola technique, in practice. Thus, the calculations and results presented by Mäkinen (1993) are correct but unnecessarily complicated, regarding the earthing impedance matrix.

Pirjola (2008) considers situations where two substations are close, so that the current to the ground from one produces a voltage drop that influences the other substation. In circuit terms, this means there is a connection between the current paths from each substation to ground. This effect is included in the Lehtinen-Pirjola method by off-diagonal elements in the impedance matrix, although, in the situations considered by Pirjola (2008), it was concluded that the offdiagonal elements do not play a major role in practice. In this paper, we have shown that an equivalent effect occurs when there are paths for GIC flows through two or more transformer windings that share a common path through the substation grounding resistance, so that current through one transformer winding produces a voltage drop that influences the current flow through the other transformer windings. This can also be modelled in the Lehtinen-Pirjola method by including off-diagonal elements in the impedance matrix. It is recommended, however, that any point at which current paths join is represented by a node in the circuit. If this is done, then there is no need for off-diagonal elements in the Lehtinen-Pirjola impedance matrix and the circuit equations can be solved using either the Nodal Admittance Matrix method or the Lehtinen-Pirjola method. This is illustrated in Fig. 8. The circuit representation in Fig. 8a is not suitable for use with the Nodal Admittance Matrix method but can be modelled by the Lehtinen-Pirjola method with the use of non-diagonal elements in the impedance matrix. However, the same circuit, with the introduction of a node at the neutral point (Fig. 8b), can be satisfactorily modelled using either the Lehtinen-Pirjola method or the Nodal Admittance Matrix method. Thus, either method can be used to model different power-system configurations, provided that the circuit model is constructed with nodes at all places that are a junction between paths where GIC flow. 
The methods described here show how GIC can be calculated for different windings of transformers. When a transformer has different values of GIC in its windings, an "effective GIC" can be calculated that gives the same degree of transformer saturation as GIC in a single winding (Albertson et al., 1981; Zheng et al., 2014). For both two-winding transformers and autotransformers, the effective GIC is given by

$I_{\mathrm{eff}}=\left|\frac{N I_{H}+I_{\mathrm{L}}}{N}\right|$,

where $N=N_{H} / N_{\mathrm{L}}=V_{H} / V_{\mathrm{L}}$ is the turns ratio of the transformer.

This is applicable to autotransformers, as illustrated in Fig. 5, or two-winding transformers, as illustrated in Fig. 4. However, Chunming Liu (personal communication, 2014) has pointed out that not all two-winding transformers have neutral points on each side that are both grounded. In such a case, the neutral points are not connected and on the ungrounded side there is no path to the ground for GIC to flow, so the GIC values in that winding will be zero.

The methodologies described above provide the techniques for modelling GIC in a power system including the GIC at different voltage levels and the GIC flow through transformer windings between voltage levels. It has been shown that the Nodal Admittance Matrix method and the Lehtinen-Pirjola method are mathematically equivalent, with both methods representing the power system as a network of admittances. The Mesh Impedance Matrix method and the Nodal Admittance Matrix method can be described as "electrically equivalent" as they are based on different circuit representations that are electrically equivalent. However, they cannot be easily compared mathematically as they are based on different numbering schemes: the Mesh Impedance Matrix method is based on loop number, while the Nodal Admittance Matrix method and Lehtinen-Pirjola method are based on node number. All the methods will give the same values for GIC and they have been tested using a benchmark model (Horton et al., 2012). The accuracy of GIC values obtained for any real power network is now dependent on the input values used for the magnetic field variations, earth conductivity structure and power-system parameters, rather than the modelling methodology.

\section{Conclusions}

It has been shown that the Nodal Admittance Matrix method used in network modelling of GIC and the Lehtinen-Pirjola method for modelling GIC are mathematically equivalent. There are three steps involved in calculating GIC using the Nodal Admittance Matrix method. The first step is to use the voltage sources in the lines representing the induced geoelectric field to calculate equivalent current sources and sum these to obtain the nodal current sources $\left[\mathbf{J}^{\mathrm{e}}\right]$. The second step is to perform the matrix inversion $[\mathbf{Y}]^{-1}$ and multiply by $\left[\mathbf{J}^{\mathrm{e}}\right]$ to obtain the nodal voltages $\left[\mathbf{V}^{n}\right]$. The third step is to use the nodal voltages to determine the currents in the lines and in the ground connections. In the Lehtinen-Pirjola method, the second and third step of the Nodal Admittance Matrix calculation are combined into one matrix expression. This involves inversion of a more complicated matrix but yields the currents to the ground $\left[\mathbf{I}^{\mathrm{e}}\right]$ directly from the nodal current sources $\left[\mathbf{J}^{\mathrm{e}}\right]$.

Accurate modelling of geomagnetically induced currents in power systems requires consideration of the GIC flows in and between different voltage levels. This can be handled by the standard Nodal Admittance Matrix and Lehtinen-Pirjola methods. GIC flows to the ground through the high-voltage and low-voltage windings of a transformer share a common path through the substation grounding resistance. This may lead to non-zero, off-diagonal elements in the earthing impedance matrix of the LP method. However, in both the Nodal Admittance Matrix method and the Lehtinen-Pirjola method, this is more easily included by introducing a node at the neutral point. It is recommended that neutral points always be included as nodes in the network model, although not required if there are only autotransformers at a substation, because this provides a more versatile and standardised modelling scheme.

Acknowledgements. This work was performed as part of Natural Resources Canada's Public Safety Geoscience program. This work benefitted from useful discussions with A. Foss, L. Trichtchenko, C. Liu and K. Zheng.

Topical Editor G. Balasis thanks S. Potirakis and J. M. Torta for their help in evaluating this paper.

\section{References}

Albertson, V. D., Kappenman, J. G., Mohan, N., and Skarbakka, G. A.: Load-Flow Studies in the Presence of GeomagneticallyInduced Currents, IEEE Trans. on Power Apparatus and Systems, PAS-100, 594-606, 1981.

Bolduc, L.: GIC observations and studies in the Hydro-Québec power system, J. Atmos. Sol.-Terr. Phy., 64, 1793-1802, 2002.

Boteler, D. H.: The Super Storms of August/September 1859 and their Effects on the Telegraph System, Adv. Space Res., 38, 159172, 2006.

Boteler, D. H.: Methodology for simulation of geomagnetically induced currents in power systems, J. Space Weather Space Climate, 4, A21, doi:10.1051/swsc/2014018, 2014.

Boteler, D. H. and Pirjola, R. J.: Modelling geomagnetically induced currents produced by realistic and uniform electric fields, IEEE Trans. Power Delivery, 13, 1303-1308, 1998.

Boteler, D. H., Pirjola, R. J., and Nevanlinna, H.: The effects of geomagnetic disturbances on electrical systems at the Earth's surface, Adv. Space Res., 22, 17-27, 1998.

Boteler, D. H., Pirjola, R., Blais, C., and Foss, A.: Development of a GIC Simulator, Paper 14PESGM1889, Proceedings of IEEE PES General Meeting, Washington, DC, July 2014. 
Caraballo, R., Sánchez Bettucci, L., and Tancredi, G.: Geomagnetically induced currents in the Uruguayan high-voltage power grid, Geophys. J. Int., 195, 844-853, 2013.

Davidson, W. F.: The magnetic storm of March 24, 1940 - Effects in the power system, Edison Electric Institute Bulletin, 365-366 and 374, July 1940.

Demiray, T., Beccuti, G., and Andersson, G.: Risk assessment of the impact of geomagnetic disturbances on the transmission grid in Switzerland, Proc. Power and Energy Society General Meeting (PES), 2013 IEEE, doi:10.1109/PESMG.2013.6672600, 2013.

Foss, A. and Boteler, D.: GIC Simulation using Network Modeling, Paper 806, Proc. Canadian Conference on Electrical and Computer Engineering (CCECE), Ottawa, Canada, 7-10 May, 4 pp., 2006.

Horton, R., Boteler, D. H., Overbye, T. J., Pirjola, R. J., and Dugan, R.: A test case for the calculation of geomagnetically induced currents, IEEE Trans. Power Delivery, 27, 2368-2373, 2012.

Jacobson, D. A. N., Shelemy, S., Chandrasena, W., Boteler, D., and Pirjola, R.: Development of Advanced GIC Analysis Tools for the Manitoba Power Grid, Submitted to CIGRE 2014, Paris, 2014.

Kappenman, J. G.: Geomagnetic Disturbances and Impacts upon Power System Operation, in: The Electric Power Engineering Handbook, edited by: Grigsby, L. L., CRC Press/IEEE Press, 2nd Edn., Chapter 16, 16.1-16.22, 2007.

Kappenman, J. G.: Geomagnetic storms and their impact on the US power grid, Report Meta-R-319 prepared for the Oak Ridge National Laboratory, USA, January 2010.

Lehtinen, M. and Pirjola, R.: Currents produced in earthed conductor networks by geomagnetically-induced electric fields, Ann. Geophys., 3, 479-484, 1985, http://www.ann-geophys.net/3/479/1985/.

Mäkinen, T.: Geomagnetically induced currents in the Finnish power transmission system, Finnish Meteorological Institute, Geophysical Publications, No. 32, Helsinki, Finland, 101 pp., 1993.

Molinski, T. S.: Why utilities respect geomagnetically induced currents, J. Atmos. Sol.-Terr. Phy., 64, 1765-1778, 2002.

Overbye, T. J., Hutchins, T. R., Shetye, K., Weber, J., and Dahman, S.: Integration of Geomagnetic Disturbance Modeling into the Power Flow: A Methodology for Large-Scale System Studies, Proc. 2012 North America Power Symposium (NAPS), Champaign, Il, 2012.
Pirjola, R.: Effects of space weather on high-latitude ground systems, Adv. Space Res., 36, 2231-2240, doi:10.1016/j.asr.2003.04.074, 2005.

Pirjola, R.: Calculation of geomagnetically induced currents (GIC) in a high-voltage electric power transmission system and estimation of effects of overhead shield wires on GIC modelling, J. Atmos. Sol.-Terr. Phy., 69, 1305-1311, 2007.

Pirjola, R.: Effects of interactions between stations on the calculation of geomagnetically induced currents in an electric power transmission system, Earth Planets Space, 60, 743-751, 2008.

Thomson, A. W. P., McKay, A. J., Clarke, E., and Reay, S. J.: Surface electric fields and geomagnetically induced currents in the Scottish Power grid during the 30 October 2003 geomagnetic storm, Space Weather, 3, S11002, doi:10.1029/2005SW000156, 2005.

Torta, J. M., Serrano, L., Regué, J. R., Sánchez, A. M., and Roldán, E.: Geomagnetically induced currents in a power grid of northeastern Spain, Space Weather, 10, S06002, doi:10.1029/2012SW000793, 2012.

Wik, M., Viljanen, A., Pirjola, R., Pulkkinen, A., Wintoft, P., and Lundstedt, H.: Calculation of geomagnetically induced currents in the $400 \mathrm{kV}$ power grid in southern Sweden, Space Weather, 6, S07005, doi:10.1029/2007SW000343, 2008.

Wik, M., Pirjola, R., Lundstedt, H., Viljanen, A., Wintoft, P., and Pulkkinen, A.: Space weather events in July 1982 and October 2003 and the effects of geomagnetically induced currents on Swedish technical systems, Ann. Geophys., 27, 1775-1787, doi:10.5194/angeo-27-1775-2009, 2009.

Zheng, K., Trichtchenko, L., Pirjola, R., and Liu, L.-G.: Effects of geophysical paramaters on GIC illustrated by benchmark network modelling, IEEE Trans. Power Delivery, 28, 1183-1191, 2013.

Zheng, K., Boteler, D. H., Pirjola, R., Liu, L. G., Becker, R., Marti, L., Boutilier, S., and Guillon, S.: Effects of System Characteristics on Geomagnetically Induced Currents, IEEE Trans. Power Delivery, 29, 890-898, 2014. 